\title{
Generic off-diagonal solutions and jet deformations in modified and/or higher dimension gravity theories
}

\author{
Subhash Rajpoot \\ California State University at Long Beach; Long Beach, California, USA \\ E-mail: Subhash.Rajpoot@csulb.edu
}

Sergiu I. Vacaru

Rector's Department, Alexandru Ioan Cuza University, UAIC

Al. Lapuşneanu str., nr. 14, UAIC - Corpus R, office 323; Iaşi, Romania, 700057; and * M. Planck Inst. Physics (W. Heisenberg Inst.), Föhringer Ring 6, München D-80805, Germany; Leibniz Univ. Hannover, Instit. Theoretical Physics, Appelstrasse 2, Hannover 30167, Germany E-mails: sergiu.vacaru@uaic.ro; Sergiu.Vacaru@gmail.com

Let $\mathbf{g}$ be a pseudo-Riemanian metric on a manifold $\mathbf{V}$ with conventional $n+n$ dimensional splitting, $\quad n \geq 2$, for a nonholonomic (non-integrable) distribution $\mathcal{N}$ and consider a correspondingly adapted linear metric compatible connection $\widehat{\mathbf{D}}$ and its torsion $\widehat{\mathcal{T}}$, both completely determined by $\mathbf{g}$. We prove that there are certain generalized frame and/or jet transforms and prolongations with $(\mathbf{g}, \mathbf{V}) \rightarrow(\widehat{\mathbf{g}}, \widehat{\mathbf{V}})$ into explicit classes of solutions of some generalized Einstein equations $\widehat{\mathbf{R}} i c=\Lambda \widehat{\mathrm{g}}, \Lambda=$ const, encoding various types of (nonholonomic) Ricci soliton configurations and/or jet variables and symmetries, in particular, subject to the condition $\overrightarrow{\mathcal{T}}=0$. This allows us to construct in general form generic off-diagonal exact solutions depending on all space time coordinates on $\mathbf{V}$ and its jet prolongations, via generating and integration functions and various classes of constants and associated symmetries. We consider an example when exact solutions are constructed as nonholonomic jet prolongations of the Kerr metrics, with possible Ricci soliton deformations, and characterized by generalized connections.

Keywords: Nonholonomic manifolds and jets; geometric methods and PDE; Ricci solitons and Einstein spaces; exact solutions and modified gravity.

\section{Nonholonomic jets and (pseudo) Riemannian manifolds}

Jets are certain equivalence classes of smooth maps between two manifolds $M, \operatorname{dim} M=n$, and $Q, \operatorname{dim} Q=m$, when maps are represented by Taylor polynomials $^{7}$. One writes this as $f, g: M \rightarrow Q:$ a $r$-jet is determined at a point $u \in M$ if there is a $r$-th order contact at $u$. Two curves $\gamma, \delta: \mathbb{R} \rightarrow V$ have the $r$-th contact at zero if for every smooth function $\varphi$ on $M$ the difference $\varphi \circ \gamma-\varphi \circ \delta$ vanishes to $r$-th order at $0 \in \mathbb{R}$. In this case, we have an equivalence relation $\gamma \sim_{r} \delta$ when $r=0$ means $\gamma(0)=\delta(0)$. If $\gamma \sim_{r} \delta$, then $f \circ \gamma \sim_{r} f \circ \delta$ for every map $f: b \rightarrow Q$.

Two maps $f, g: V \rightarrow Q$ are said to determine the same $r$-jet at $x \in M$, if for every curve $\gamma: \mathbb{R} \rightarrow V$ with $\gamma(0)=x$ the curves $f \circ \gamma$ and $g \circ \gamma$ have the $r$-th order contact at zero. In such a case, we write $j_{x}^{r} f=j_{x}^{r} g$, or $j^{r} f(x)=j^{r} g(x)$. An equivalence class of this relation is called an $r$-jets of $M$ into $Q$. The set of all $r$-jets of $M$ into $Q$ is denoted by $J^{r}(M, Q)$; for an element $X=j_{x}^{r} f \in J^{r}(M, Q)$, the point $x:=\alpha X$ is the source of $X$ and the point $f(x)=: \beta X$ is the target of $X$.

*DAAD fellowship affiliations for two host institutions 
One denotes by $\pi_{s}^{r}, 0 \leq s \leq r$ the projection $j_{x}^{r} f \rightarrow j_{x}^{s} f$ of $r$-jets into $s$-jets. All $r$-jets form a category, the units of which are the $r$-jets of the identity maps of manifolds. By $J_{x}^{r}(M, Q)$, or $J_{x}^{r}(M, Q)_{y}$, we mean the set of all $r$-jets of $x$ onto $Q$ with source $x \in M$, or tangent $y \in Q$, respectively, and we write $J_{x}^{r}(M, Q)_{y}=$ $J_{x}^{r}(M, Q) \cap J_{x}^{r}(M, Q)_{y}$ and $L_{n, m}^{r}=J_{0}^{r}\left(\mathbb{R}^{n}, \mathbb{R}^{m}\right)_{0}$. In local coordinates $x^{i}$, we write $\partial_{i} f:=\frac{\partial^{|\bar{i}|} f}{\left(\partial x^{1}\right)^{i_{1}} \ldots\left(\partial x^{n}\right)^{i n}}$ as the partial derivative of a function $f: U \subset \mathbb{R}^{n} \rightarrow \mathbb{R}$, with a multi-index $\breve{i}$ of range $n$, which is a $m$-tuple $\breve{i}=\left(i_{1}, \ldots, i_{n}\right)$ of non-negative integers. We use $|\check{i}|=i_{1}+\ldots+i_{n}$, with $\check{i} !=i_{1} ! i_{2} ! \ldots i_{n} !, 0 !=1$, and $x^{\breve{i}}=\left(x^{1}\right)^{i_{1}} \ldots\left(x^{n}\right)^{i_{n}}$ for $x=\left(x^{1}, x^{2}, \ldots, x^{n}\right) \in \mathbb{R}^{n}$.

In this work, we study nonholonomic jet prolongations of the geometric objects in $\mathbf{J}^{r}\left(\mathbf{V}, \mathbf{V}^{\prime}\right)$-framework, where boldface letters are used for spaces and objects adapted to certain non-integrable constraints, with local coordinates $u^{\alpha_{s}}=$ $\left(x^{i}, y^{a}, \zeta_{\check{\alpha}_{1} \ldots \check{\alpha}_{r}}^{\alpha^{\prime}}\right)=\left(x^{i}, y^{a}, \zeta^{a_{s}}\right)$. We use the label $s$ in order to perform a conventional splitting of dimensions, $\operatorname{dim}{ }^{s} V=4+2 s=2+2+\ldots+2 \geq 4 ; s \geq 0$ for conventional finite dimensional (pseudo) Riemannian space ${ }^{s} V$. The jet coordinates $v_{\check{\alpha}_{1} \ldots \check{\alpha}_{r}}^{\alpha^{\prime}}$ are re-grouped in oriented two shells which allows us to apply the AFDM and to construct exact solutions for generalized Einstein equations and metrics ${ }^{s} \mathbf{g}$ with arbitrary signatures $( \pm 1, \pm 1, \pm 1, \ldots \pm 1)$. Such shells are determined by nonholonomic data which transforms into $\zeta_{\check{\alpha}_{1} \ldots \check{\alpha}_{r}}^{\alpha^{\prime}}$ with symmetric low indices if the constructions are performed in coordinate bases. Let us establish conventions on (abstract) indices and coordinates $u^{\alpha_{s}}=\left(x^{i_{s}}, y^{a_{s}}\right)$, for $s=0,1,2,3, \ldots$ labelling the oriented number of two dimensional, 2-d, "shells" added to a 4-d spacetime. For $s=0$ (in a conventional form), we write $u^{\alpha}=\left(x^{i}, y^{a}\right)$ and consider such local systems of coordinates: $s=1: u^{\alpha_{1}}=\left(x^{\alpha}=u^{\alpha}, v^{a_{1}}\right)=\left(x^{i}, y^{a}, \zeta^{a_{1}}\right) ; s=2: u^{\alpha_{2}}=\left(x^{\alpha_{1}}=u^{\alpha_{1}}, v^{a_{2}}\right)=$ $\left(x^{i}, y^{a}, \zeta^{a_{1}}, \zeta^{a_{2}}\right) ; s=3: u^{\alpha_{3}}=\left(x^{\alpha_{2}}=u^{\alpha_{2}}, v^{a_{3}}\right)=\left(x^{i}, y^{a}, \zeta^{a_{1}}, \zeta^{a_{2}}, \zeta^{a_{3}}\right), \ldots$ for $i, j, \ldots=1,2 ; a, b, \ldots=3,4 ; a_{1}, b_{1} \ldots=5,6 ; a_{2}, b_{2} \ldots=7,8 ; a_{3}, b_{3} \ldots=9,10, \ldots$ and $i_{1}, j_{1}, \ldots=1,2,3,4 ; i_{2}, j_{2}, \ldots=1,2,3,4,5,6 ; i_{3}, j_{3}, \ldots=1,2,3,4,5,6,7,8 ; \ldots$ In brief, we write $u=(x, y) ;{ }^{1} u=\left(u,{ }^{1} \zeta\right)=\left(x, y,{ }^{1} \zeta\right),{ }^{2} u=\left({ }^{1} u,{ }^{2} \zeta\right)=\left(x, y,{ }^{1} \zeta,{ }^{2} \zeta\right), \ldots$

\section{Jet prolongated Ricci soliton and Einstein equations}

A map $j^{r} f: M \rightarrow J^{r}(M, Q)$ is called a $r$-th jet prolongation of $f: M \rightarrow Q$. The set $J^{r} Q$ of all $r$-jets of the local sections of $Y$ is called the $r$-th jet prolongation of $Q$ and $J^{r} Q \subset J^{r}(M, Q)$ is a closed submanifold. Any $\mathbf{N}$-connection structure $\mathbf{N}$ on $\mathbf{V}$ determines a $r$-th jet prolongated $\mathbf{N}$-connection ${ }^{s} \mathbf{N}$ on $\mathbf{J}^{r}\left(\mathbf{V}, \mathbf{V}^{\prime}\right)$ as Whitney sum

$$
{ }^{s} \mathbf{N}: T{ }^{s} \mathbf{V}=h \mathbf{V} \oplus v \mathbf{V} \oplus{ }^{1} v \mathbf{V} \oplus{ }^{2} v \mathbf{V} \oplus \ldots \oplus{ }^{s} v \mathbf{V}
$$

for a conventional horizontal (h) and vertical (v) "shell by shell" splitting. $r$-th jet prolongations induce on $\mathbf{J}^{r}\left(\mathbf{V}, \mathbf{V}^{\prime}\right)$ a system of N-elongated bases/ partial derivatives, $\mathbf{e}_{\nu_{s}}=\left(\mathbf{e}_{i_{s}}, e_{a_{s}}\right)$, and N-adapted differentials, $\mathbf{e}^{\mu_{s}}=\left(e^{i_{s}}, \mathbf{e}^{a_{s}}\right)$, with $\mathbf{e}_{i_{s}}=\frac{\partial}{\partial x^{i_{s}}}-N_{i_{s}}^{a_{s}} \partial_{a_{s}}, e_{a_{s}}=\partial_{a_{s}}=\frac{\partial}{\partial \zeta^{a_{s}}}, e^{i_{s}}=d x^{i_{s}}, \mathbf{e}^{a_{s}}=d \zeta^{a_{s}}+N_{i_{s}}^{a_{s}} d x^{i_{s}}$, 
satisfying anholonomy relations

$$
\left[\mathbf{e}_{\alpha_{s}}, \mathbf{e}_{\beta_{s}}\right]=\mathbf{e}_{\alpha_{s}} \mathbf{e}_{\beta_{s}}-\mathbf{e}_{\beta_{s}} \mathbf{e}_{\alpha_{s}}=W_{\alpha_{s} \beta_{s}}^{\gamma_{s}} \mathbf{e}_{\gamma_{s}},
$$

when $W_{i_{s} a_{s}}^{b_{s}}=\partial_{a_{s}} N_{i_{s}}^{b_{s}}$ and $W_{j_{s} i_{s}}^{a_{s}}={ }^{J} N_{i_{s} j_{s}}^{a_{s}}$, where the Neijenhuis tensor, i.e. the curvature of the $r$-th jet prolongated $\mathrm{N}$-connection, is

$$
{ }^{J} N_{i_{s} j_{s}}^{a_{s}}=\mathbf{e}_{j_{s}}\left(N_{i_{s}}^{a_{s}}\right)-\mathbf{e}_{i_{s}}\left(N_{j_{s}}^{a_{s}}\right) .
$$

On $\mathbf{J}^{r}\left(\mathbf{V}, \mathbf{V}^{\prime}\right)$ with prolongation of geometric objects from $\mathbf{V}$, we define linear connection structures in $\mathrm{N}$-adapted form. There are distinguished connection, $\mathrm{d}-$ connection, structures,

$$
\begin{aligned}
{ }^{s} \mathbf{D} & =\left\{D_{\alpha_{s}}\right\}, \mathbf{D}=(h D ; v D),{ }^{1} \mathbf{D}=\left({ }^{1} h D ;{ }^{1} v D\right), \ldots, \\
{ }^{s-1} \mathbf{D} & =\left({ }^{s-2} h D ;{ }^{s-1} v D\right),{ }^{s} \mathbf{D}=\left({ }^{s-1} h D ;{ }^{s} v D\right),
\end{aligned}
$$

preserving under parallelism the $\mathrm{N}$-connection splitting. There are natural $r$-th jet prolongations of the torsion, $\mathcal{T}^{\alpha_{s}}=\left\{\mathbf{T}_{\beta_{s} \gamma_{s}}^{\alpha_{s}}\right\}$, and curvature, $\mathcal{R}_{\beta_{s}}^{\alpha_{s}}=\left\{\mathbf{R}_{\beta_{s} \gamma_{s} \delta_{s}}^{\alpha_{s}}\right.$, tensors ${ }^{s} \mathbf{D}$ defined on a prime $\mathbf{V}$ and elongated in $\mathrm{N}$-adapted form on $\mathbf{J}^{r}\left(\mathbf{V}, \mathbf{V}^{\prime}\right)$. In standard $r$-jet coordinates for $\mathbf{J}^{r}\left(\mathbf{V}, \mathbf{V}^{\prime}\right), u^{\underline{\alpha}_{s}}=\left(x^{i}, y^{a}, \zeta_{\check{\alpha}_{1} \ldots \check{\alpha}_{r}}^{\alpha^{\prime}}\right)$, additional contraction of up-down indices and symmetrization result in very cumbersome coefficient formulas. A metric structure on ${ }^{s} \mathbf{V}$ can be written as a distinguished metric

$$
{ }^{s} \mathbf{g}=g_{i_{s} j_{s}}\left({ }^{s} u\right) e^{i_{s}} \otimes e^{j_{s}}+g_{a_{s} b_{s}}\left({ }^{s} u\right) \mathbf{e}^{a_{s}} \otimes \mathbf{e}^{b_{s}} .
$$

In nonholonomic $\mathrm{N}$-adapted $r$-jet variables on natural prolongations from $\mathbf{V}$ on $\mathbf{J}^{r}\left(\mathbf{V}, \mathbf{V}^{\prime}\right)$, the gradient canonical Ricci jet-solitons are defined by equations ${ }^{4}$

$$
\widehat{\mathbf{R}}_{\beta_{s} \gamma_{s}}+\widehat{\mathbf{D}}_{\beta_{s}} \widehat{\mathbf{D}}_{\gamma_{s}} \kappa=\lambda \mathbf{g}_{\beta_{s} \gamma_{s}} .
$$

Our goal is to elaborate on a geometric method for decoupling the equations (1), which for certain classes of nonholonomic constraints transforms into systems of nonlinear PDE with possible zero torsion conditions. We are able to find general classes of solutions when $\kappa$ is parameterized in such forms that ${ }^{3-7}$

$$
\begin{array}{cc}
\widehat{\mathbf{R}}_{i j}={ }^{h} \Upsilon\left(x^{k}\right) \mathbf{g}_{i j}, & \\
\widehat{\mathbf{R}}_{a b}={ }^{v} \Upsilon\left(x^{k}, y^{a}\right) \mathbf{g}_{a b}, \text { for jet shells: } & \widehat{\mathbf{R}}_{a_{s} b_{s}}={ }^{s}{ }^{v} \Upsilon\left(x^{k}, y^{a}, \zeta^{a_{1}}, \ldots, \zeta^{a_{s}}\right) \mathbf{g}_{a_{s} b_{s}}, \\
\widehat{\mathbf{R}}_{\beta \gamma}=0, \text { for } \beta \neq \gamma, & \widehat{\mathbf{R}}_{\beta_{s} \gamma_{s}}=0, \text { for } \beta_{s} \neq \gamma_{s} .
\end{array}
$$

\section{Exact solutions and nonholonomic jet prolongations}

The Boyer-Linquist coordinates for the Kerr metric were introduced as $(r, \vartheta, \varphi, t)$, where $r=m_{0}\left(1+p \widehat{x}_{1}\right), \widehat{x}_{2}=\cos \vartheta$. The parameters $p, q$ are related to the total black hole mass, $m_{0}$ and the total angular momentum, $a m_{0}$, for the asymptotically flat, stationary and axisymmetric Kerr spacetime. The formulas $m_{0}=M p^{-1}$ and $a=M q p^{-1}$ when $p^{2}+q^{2}=1$ implies $m_{0}^{2}-a^{2}=M^{2}$, resulting in

$$
\begin{aligned}
d s_{[0]}^{2} & =\left(d x^{1^{\prime}}\right)^{2}+\left(d x^{2^{\prime}}\right)^{2}+\bar{A}\left(\mathbf{e}^{3^{\prime}}\right)^{2}+\left(\bar{C}-\bar{B}^{2} / \bar{A}\right)\left(\mathbf{e}^{4^{\prime}}\right)^{2}, \\
\mathbf{e}^{3^{\prime}} & =d t+d \varphi \bar{B} / \bar{A}=d y^{3^{\prime}}-\partial_{i^{\prime}}\left(\widehat{y}^{3^{\prime}}+\varphi \bar{B} / \bar{A}\right) d x^{i^{\prime}}, \mathbf{e}^{4^{\prime}}=d y^{4^{\prime}}=d \varphi,
\end{aligned}
$$


where $x^{1^{\prime}}(r, \vartheta), x^{2^{\prime}}(r, \vartheta), y^{3^{\prime}}=t+\widehat{y}^{3^{\prime}}(r, \vartheta, \varphi)+\varphi \bar{B} / \bar{A}, y^{4^{\prime}}=\varphi, \partial_{\varphi} \widehat{y}^{3^{\prime}}=-\bar{B} / \bar{A}$, for which

$$
\left(d x^{1^{\prime}}\right)^{2}+\left(d x^{2^{\prime}}\right)^{2}=\Xi\left(\Delta^{-1} d r^{2}+d \vartheta^{2}\right),
$$

when the coefficients are

$$
\begin{aligned}
& \bar{A}=-\Xi^{-1}\left(\Delta-a^{2} \sin ^{2} \vartheta\right), \bar{B}=\Xi^{-1} a \sin ^{2} \vartheta\left[\Delta-\left(r^{2}+a^{2}\right)\right], \\
& \bar{C}=\Xi^{-1} \sin ^{2} \vartheta\left[\left(r^{2}+a^{2}\right)^{2}-\Delta a^{2} \sin ^{2} \vartheta\right], \\
& \Delta=r^{2}-2 m_{0}+a^{2}, \Xi=r^{2}+a^{2} \cos ^{2} \vartheta .
\end{aligned}
$$

Kerr Ricci soliton deformations and effective vacuum $r-$ jet prolongations: There are classes of solutions with jet variables describing vacuum ellipsoid spacetime configurations prolongated on two shell jet variables when the source is of type $\Upsilon=\widetilde{\lambda}+\varepsilon(\widetilde{\Lambda}+\Lambda)=0$, with effective gravity mass term ${ }^{\mu} \tilde{\Lambda}=\mu_{g}^{2}|\lambda|$, resulting in ellipsoidal off-diagonal configurations in GR. For such metrics, $\varepsilon=-{ }^{\mu} \tilde{\Lambda} /(\widetilde{\Lambda}+\Lambda) \ll 1$ can be considered as an eccentricity parameter. The corresponding models of offdiagonal jet interior gravitational interactions are with $f$-modifications when $\widetilde{\Lambda}$ compensates nonholonomic contributions via effective constant $\widetilde{\Lambda}$ and relates the constructions to massive gravity deformations of a Kerr solution. This subclass of solutions for $\varepsilon$-deformations into vacuum solutions is parameterized by

$$
\begin{aligned}
d s^{2}= & e^{\psi\left(x^{k^{\prime}}\right)}\left(1+\varepsilon \chi\left(x^{k^{\prime}}\right)\right)\left[\left(d x^{1^{\prime}}\right)^{2}+\left(d x^{2^{\prime}}\right)^{2}\right] \\
& -\frac{\tilde{\Phi}^{2} \bar{A}}{4 \mu \tilde{\Lambda}}\left[1+\varepsilon \chi_{3^{\prime}}\right]\left[d y^{3^{\prime}}+\left(\partial_{k^{\prime}}{ }^{\eta} n\left(x^{i^{\prime}}\right)-\partial_{k^{\prime}}\left(\widehat{y}^{3^{\prime}}+\varphi \bar{B} / \bar{A}\right)\right) d x^{k^{\prime}}\right]^{2} \\
& +\frac{\left(\partial_{\varphi} \tilde{\Phi}\right)^{2} \eta_{4^{\prime}}}{\mu \tilde{\Lambda} \tilde{\Phi}^{2}}\left(\bar{C}-\frac{\bar{B}^{2}}{\bar{A}}\right)\left[1+\varepsilon \chi_{4^{\prime}}\right]\left[d \varphi+\left(\partial_{i^{\prime}} \widetilde{A}+\varepsilon \partial_{i^{\prime}}{ }^{1} \check{A}\right) d x^{i^{\prime}}\right]^{2} \\
& +\frac{{ }^{1} \tilde{\Phi}^{2}}{4(\widetilde{\Lambda}+\Lambda)}\left[d \zeta^{5}+\left(\partial_{\tau}{ }^{1} n\right) d u^{\tau}\right]^{2}+\frac{\left(\widetilde{\partial}_{6}{ }^{1} \tilde{\Phi}\right)^{2}}{(\widetilde{\Lambda}+\Lambda)^{1} \tilde{\Phi}^{2}}\left[d \zeta^{6}+\left(\partial_{\tau}{ }^{1} \check{A}\right) d u^{\tau}\right]^{2} \\
& +\frac{{ }^{2} \tilde{\Phi}^{2}}{4(\widetilde{\Lambda}+\Lambda)}\left[d \zeta^{7}+\left(\partial_{\tau_{1}}{ }^{2} n\right) d u^{\tau_{1}}\right]^{2}+\frac{\left(\widetilde{\partial}_{8}{ }^{2} \tilde{\Phi}\right)^{2}}{(\widetilde{\Lambda}+\Lambda)^{2} \tilde{\Phi}^{2}}\left[d \zeta^{8}+\left(\partial_{\tau_{1}}{ }^{2} \check{A}\right) d u^{\tau_{1}}\right]^{2} .
\end{aligned}
$$

The jet components are generated by functions ${ }^{1} \tilde{\Phi},{ }^{2} \tilde{\Phi}$ and $\mathrm{N}$-coefficients modified effective jet prolongated sources, $\Lambda \rightarrow \widetilde{\Lambda}+\Lambda$. This result shows that interior jet interactions can mimic $\varepsilon$-deformations in order to compensate contributions from $f$-modifications and even effective vacuum configurations for the $4-\mathrm{d}$ horizontal part ${ }^{1,2,4}$. In general, such effective vacuum metrics encode possible jet modifications/ polarizations of physical constants and coefficients of metrics under nonlinear polarizations of an effective 8-d vacuum distinguishing 4-d nonholonomic configurations and Ricci soliton or massive gravity contributions ${ }^{7}$. Jet variables and $f$-modified contributions are described by terms proportional to eccentricity $\varepsilon$.

Future research on extended on jet variables (super) gravity and cosmological solutions in modified gravity theories a planned following recent works on supersting gravity and modified gravity theories ${ }^{8,9}$. 


\section{Acknowledgments}

$\mathrm{SV}$ is supported by a travel grant from MG14 and reports certain research related to his basic activity at UAIC, a DAAD fellowship and the Program IDEI, PN-II-IDPCE-2011-3-0256. He is grateful for DAAD hosting to D. Lüst and O. Lechtenfeld.

\section{References}

1. S. Vacaru, J. Math. Phys. 46, 042503 (2005); arXiv: gr-qc/0307103.

2. S. Vacaru, Class. Quant. Grav. 28, 215991 (2011); arXiv: 1008.4912.

3. E. Guendelman, H. Nishino, S. Rajpoot, Phys. Rev. D 87, 027702 (2013).

4. T. Gheorghiu, O. Vacaru, S. Vacaru, EPJC 74, 3152 (2014); arXiv: 1312.4844.

5. E. Elizalde, S. Vacaru, Gen. Relat. Grav. 47, 64 (2015).

6. S. Vacaru, Mod. Phys. Lett. A 30, nr. 19, 1550090 (2015); arXiv: 1308.6180.

7. S. Rajpoot and S. Vacaru, Nonholonomic Jet Deformations and Exact Solutions for Modified Ricci Soliton and Einstein Equations, arXiv: 1411.1861.

8. T. Gheorghiu, O. Vacaru, S. Vacaru, Class. Quant. Gr. 32, 065004 (2015); arXiv: 1311.6365.

9. S. Vacaru, Phys. Lett. B 752, 27 (2016); arXiv: 1304.1080. 